\title{
Sistema de crecimiento y rendimiento maderable para plantaciones de teca (Tectona grandis L. f.) en Campeche, México
}

\author{
Growth and yield system for teak plantations (Tectona grandis L. F.) in \\ Campeche, Mexico
}

\section{Juan Carlos Tamarit-Urias*, Héctor Manuel De los Santos-Posadas², Arnulfo Aldrete², José René Valdez-Lazalde², Hugo Ramírez-Maldonado ${ }^{3}$ y Vidal Guerra-De la Cruz ${ }^{4}$}

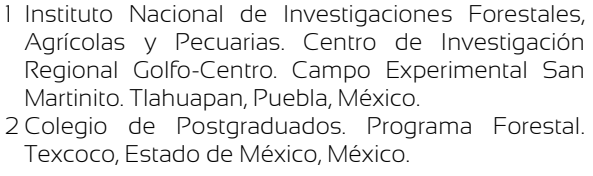
Agrícolas y Pecuarias. Centro de Investigación Regional Golfo-Centro. Campo Experimental San Martinito. Tlahuapan, Puebla, México.

2 Colegio de Postgraduados. Programa Forestal. Texcoco, Estado de México, México.

\author{
3 Universidad Autónoma Chapingo. División de \\ Ciencias Forestales. Texcoco, Estado de México, \\ México \\ 4 Instituto Nacional de Investigaciones Forestales, \\ Agrícolas y Pecuarias. Centro de Investigación \\ Regional Centro. Sitio Experimental Tlaxcala. \\ Tlaxcala, México. \\ * Autor de correspondencia. tamarit.juan@inifap.gob.mx
}

\section{RESUMEN}

Los modelos dinámicos de crecimiento y rendimiento maderable son importantes para tomar decisiones en el manejo forestal. Estos modelos predicen la dinámica futura en función de atributos del rodal y son herramientas prácticas para aplicar silvicultura en la planeación del manejo de bosques naturales y plantaciones. En México, teca (Tectona grandis L. f.) es de importancia maderable en plantaciones comerciales; sin embargo, las herramientas de este tipo para el manejo técnico y científico son limitadas. Se desarrolló un sistema de crecimiento y rendimiento maderable (Scrm) explícito para plantaciones de teca establecidas en Campeche, México. El Scrm se conformó de ecuaciones dinámicas de diferencia algebraica ajustadas al utilizar información dasométrica de una red de parcelas permanentes con remediciones. La selección de cada componente del Scrm se basó en un análisis de la bondad de ajuste y en una inspección gráfica para comparar la similitud entre el patrón generado y la tendencia observada, se buscó un balance entre consideraciones estadísticas y tendencias biológicas de crecimiento. La mortalidad se determinó a través de la densidad de plantación inicial y fue sensible al índice de sitio. Se seleccionaron sistemas de crecimiento tipo Schumacher para el área basal y el volumen. A la edad del turno planeado a 19 años y con un índice de sitio de $18 \mathrm{~m}$, se estimaron rendimientos de $152.75 \mathrm{~m}^{3} \mathrm{ha}^{-1}, 136.86 \mathrm{~m}^{3} \mathrm{ha}^{-1}$ y $113.12 \mathrm{~m}^{3} \mathrm{ha}^{-1}$ para toda la plantación en general, para la densidad de 1250 plantas por hectárea $\left(\mathrm{pl} \mathrm{ha}^{-1}\right)$ y para la densidad de $816 \mathrm{pl} \mathrm{ha}^{-1}$, respectivamente. El Scrm generado permite estimar el volumen total con corteza por hectárea bajo las condiciones locales específicas de establecimiento, de manejo silvícola y de calidad de sitio de las plantaciones de teca analizadas.

PALABRAS CLAVE: área basal, modelos de predicción y proyección, mortalidad, teca, volumen.

\section{ABSTRACT}

Forest management decisions are addressed using dynamic growth and yield equations, which predict the future forest dynamic as a function of stand-level attributes and providing information for decision-making in sustainable development. They are more widely used as practical tools in forestry and can be desirable for plantation management-planning. Teak (Tectona grandis L. f.) is an important commercial species in plantations from Mexico, although there is a lack of scientific knowledge as regards the management or modeling tools to support functional forest management decision making. In this study an explicit growth and yield system (G\&YS) was developed for plantation of Teak in Campeche, Mexico. The G\&YS was integrated for algebraic difference dynamic equations and they were fitted to pairs of dasometric variables of stem-mapped re-measurements plots. The G\&YS components were selected according to the goodness-of-fit system, also the graphical analysis between actual and fitted values was used. Both analyses considered the statistic accuracy and biologically realistic predictions. The mortality function was associated with the initial planting density and also it was sensitive to the site index. The selected growth systems for the basal area and volume per hectare were based on Schumacher equation. For a site index of $18 \mathrm{~m}$ at a reference age equal to 7.5 years and forest rotation equal to 19-year, the global estimated yield was of 152.75 $\mathrm{m}^{3} \mathrm{ha}^{-1}$, while for densities of 1,250 trees per hectare and 816 trees per hectare, the yields were $136.86 \mathrm{~m}^{3} \mathrm{ha}^{-1}$ and $113.12 \mathrm{~m}^{3} \mathrm{ha}^{-1}$, respectively. The G\&YS generated allows estimating the total volume with bark per hectare under the specific local conditions of establishment, silvicultural management and site quality of the teak plantations analyzed.

KEYWORDS: basal area, prediction and projection equations, mortality, teak, volume. 


\section{INTRODUCCIÓN}

Tectona grandis L. f. (teca) es una especie forestal maderable que se planta a gran escala en los trópicos del mundo, la Organización de las Naciones Unidas para la Agricultura y la Alimentación (FAO, por sus siglas en inglés) (2010) registró para el año 2010 una superficie plantada en el mundo de 4.3 millones de hectáreas, lo que representó $2 \%$ de la superficie mundial plantada; de esta superficie, $3 \%$ se ubicaba en regiones tropicales de América (Kollert y Cherubini, 2012). En México, para el año 2014, la superficie plantada de teca alcanzó 25324 ha que representó el tercer lugar en importancia con una tendencia creciente (Comisión Nacional Forestal [Conafor], 2014). Su madera tiene alta demanda en el mercado internacional gracias a sus excelentes propiedades tecnológicas, es considerada por algunos expertos como la madera más valiosa del mundo (Hallett, Díaz-Calvo, Villa-Castillo y Wagner, 2011; Camacho-Linton, Ramírez-Maldonado, De los SantosPosadas y Zamudio, 2013).

$\mathrm{Al}$ iniciar el siglo XXI, México ha buscado reactivar su industria forestal fomentando el establecimiento de plantaciones forestales comerciales (PFC) a escala industrial con especies de rápido crecimiento. Actualmente, la mayoría de las plantaciones de teca se encuentran en etapa de desarrollo. Esta situación demanda generar con urgencia herramientas técnicas para estimar su crecimiento y rendimiento maderable que faciliten la toma de decisiones informadas para su adecuado manejo técnico y aplicación de silvicultura cuantitativa. Estas herramientas deben desarrollarse considerando las condiciones edáficas, climáticas y silvícolas particulares de las regiones en que las PFC están establecidas.

La cuantificación del volumen maderable por unidad de superficie es la variable de mayor interés para el administrador e inversionista forestal debido a la alta importancia económica que representa. El volumen por hectárea es función de otras variables de totalidad del rodal como altura dominante, densidad, área basal, entre otras, que en combinación conforman un sistema de crecimiento y rendimiento maderable (Scrm) (Clutter, Fortson, Pienaar,
Brister y Bailey, 1983; Weiskittel, Hann, Kershaw y Vanclay, 2011; Santiago-García et al., 2015; Návar et al., 2016).

En particular, la altura dominante es útil para caracterizar la productividad forestal a determinada edad mediante el índice de sitio (IS), es una de las variables menos afectadas por cambios en la densidad y por tratamientos silvícolas; un modelo de crecimiento en altura dominante y su correspondiente expresión de IS permite estimar tal productividad y ayuda a definir el régimen de manejo silvícola, por tanto, es un indicador sencillo y fácil de evaluar, además de que es un atributo fijo y estable a lo largo del tiempo en un rodal (Pretzsch, 2009).

La forma de estimar el volumen como un grupo de variables interrelacionadas es más fiable, ya que en las variables involucradas se trabaja con modelos dinámicos de crecimiento que permiten realizar simulaciones ante diferentes alternativas de manejo silvícola y evaluar su efecto en términos de crecimiento y rendimiento (Torres y Magaña, 2005). Los Scrm se recomiendan para el manejo de las PFC, debido a que representan un compromiso entre generalidad de aplicación y precisión estadística, además, son de utilidad práctica debido a que son relativamente sencillos de implementar (Pretzsch, 2009; Parra-Piedra, De los Santos-Posadas, Fierros-González, Valdez-Lazalde y Romo-Lozano, 2017; Fierros-Mateo, De los SantosPosadas, Fierros-González y Cruz-Cobos, 2017).

A pesar de la importancia de los Scrm, la generación de este tipo de herramientas cuantitativas es limitada para las PFC de teca en México; algunos estudios en esta materia y para esta especie son el de Camacho-Linton et al., (2013), quienes a partir de información de parcelas temporales y bajo el enfoque de árbol medio sin considerar el efecto de mortalidad, elaboraron tablas de producción para PFC de Campeche. Por su parte, Tamarit et al. (2014a), a partir de información de árboles individuales y un modelo de ahusamiento de tipo segmentado, generaron un sistema de cubicación para modelar el perfil fustal y estimar tanto el volumen comercial como el fustal para PFC de Campeche, Tabasco y Chiapas; Tamarit et al. (2014b) desarrollaron ecuaciones dinámicas de altura dominante e IS utilizando datos de parcelas permanentes para clasificar la produc- 
tividad en plantaciones de Campeche. Telles, Gómez, Alanís, Aguirre y Jiménez (2018) ajustaron un modelo para estimar el volumen fustal para PFC en Michoacán.

La generación y actualización de un Scrm para una región especifica que integre como componentes los modelos resultantes de estudios previos, dará mayor certidumbre a las proyecciones de los rendimientos que se estimen. Un Scrm es una importante herramienta cuantitativa, que permite al gerente forestal diseñar estrategias de manejo silvícola y le apoya en la toma de decisiones en la administración técnica de las plantaciones.

\section{OBJETIVOS}

El objetivo fue desarrollar un sistema de crecimiento y rendimiento maderable ( $\mathrm{Scrm}$ ) explícito integrado por modelos de altura dominante, mortalidad y área basal, con base en ecuaciones dinámicas de diferencia algebraica, para estimar el volumen por hectárea a escala global y diferenciar tanto por densidad de plantación inicial como por sitio de plantación para Tectona grandis L. f. (teca) establecida en Campeche, México.

\section{MATERIALES Y MÉTODOS}

A partir de una red de 388 parcelas permanentes con remediciones, se analizó información dasométrica de plantaciones de teca establecidas en el Valle de Edzná del municipio de Campeche, México. El clima de la zona de estudio corresponde a un cálido subhúmedo con lluvias en verano, temperatura promedio anual de $26.6{ }^{\circ} \mathrm{C}$, precipitación promedio anual de $1094.7 \mathrm{~mm}$, con seis meses de sequía de diciembre a mayo y ocurrencia de vientos dominantes en invierno y verano con ráfagas máximas de hasta $60 \mathrm{~km} \mathrm{~h}^{-1}$ (Breña, 2004).

Se procesó una base de datos con mediciones e información de altura total de los árboles (At) en metros, diámetro normal (Dn) de los árboles en centímetros y la densidad de árboles vivos por parcela $(\mathrm{N})$, además del registro de la edad (E) de la plantación en meses. Se trabajó con plantaciones distribuidas en cuatro diferentes sitios de plantación identificados como I, II, III y IV, establecidas con la misma fuente de semilla y con técnicas similares de preparación del sitio, método de plantación y técnicas de manejo silvícola. Las densidades iniciales fueron de 1250 plantas ha-1 y $816 \mathrm{pl} \mathrm{ha}^{-1}$, con espaciamientos de $4 \mathrm{~m} \times 2 \mathrm{~m}$ y $3.5 \mathrm{~m} \times 3.5 \mathrm{~m}$, respectivamente.

Las parcelas se establecieron cubriendo la totalidad de las condiciones de plantación en términos de edad, densidad y calidad de sitio. La superficie de las parcelas fue de $800 \mathrm{~m}^{2}$ y $1225 \mathrm{~m}^{2}$ para el menor y mayor espaciamiento, respectivamente, abarcando 100 árboles por parcela. Las mediciones se efectuaron anualmente a partir del primer año del establecimiento de la plantación. La edad de plantación varió de 12 meses a 96 meses, para las diferentes parcelas se tuvieron de uno a nueve registros de mediciones repetidas. La información se trabajó como datos de tipo longitudinal con observaciones irregularmente espaciadas en el tiempo.

A partir de la información dasométrica básica se generaron las variables de totalidad de rodal siguientes: la altura dominante $(A)$, el número total de árboles $(N)$, el área basal total $(B)$ y el volumen fustal total $(V)$ para cada parcela, posteriormente se proyectaron valores por hectárea. Para generar modelos de predicción y proyección mediante ecuaciones dinámicas en diferencia algebraica, se conformó una base de datos bajo un arreglo en pares no traslapados. Para estimar el volumen fustal total por árbol individual (v) se usó el modelo de Schumacher-Hall generado por Tamarit et al. (2014a) para esta especie. Para estimar $A$ y el índice de sitio (IS) se usó la ecuación dinámica generada por Tamarit et al. (2014b) para esta especie, a partir del modelo base de Champan-Richards para la plantación global y por sitio de plantación, misma que fue considerada como el primer componente del Scrm, cuya estructura tuvo la forma general: $A=f(E), N=$ $g(E, A), B=h(E, A, N)$ y $V=j(E, A, N, B)$, en donde $f$, $g, h$ y $j$ son variables genéricas que indican función.

Para generar los modelos de mortalidad, los datos del número de árboles por hectárea se agruparon por densidad de plantación inicial y se evaluó el ajuste de modelos de mortalidad expresados en diferencia algebraica derivados de ecuaciones diferenciales sugeridos por Rose, Clutter, Shiver, Hall y Borders (2004); Diéguez-Aranda, Castedo- 
Dorado, Álvarez-González y Rodríguez-Soalleiro (2005); Zhao, Borders, Wang y Kane (2007) (Tabla 1).

Para el área basal en metros cuadrados por hectárea y el volumen en metros cúbicos por hectárea se construyeron y evaluaron seis sistemas de crecimiento conformados cada uno por un modelo de predicción y uno de proyección (Tabla 2); como base se utilizó el modelo tipo Schumacher (Schumacher, 1939) que inicialmente se desarrolló para relacionar el volumen con la edad. Los parámetros del modelo base se relacionaron con la altura dominante y con el número de árboles tanto en la asíntota como en la tasa de crecimiento. En todos los casos, los respectivos modelos de proyección se obtuvieron de aplicar el método de diferencia algebraica (Clutter et al., 1983) a partir del parámetro asintótico $C_{0}$ y $\mathrm{D}_{0}$ considerados como libres y que teóricamente dependen de la calidad de estación.

TABLA 1. Modelos de mortalidad evaluados y expresados en diferencia algebraica.

\begin{tabular}{ll}
\hline \multicolumn{1}{c}{ Expresión en diferencia algebraica } & Modelo \\
\hline$N_{2}=N_{1} e^{b_{1}\left(E_{2}-E_{1}\right)}$ & 1 \\
$N_{2}=\left(N_{1}^{b_{1}}+b_{2}\left(E_{2}-E_{1}\right)\right)^{1 / b_{1}}$ & 3 \\
$N_{2}=N_{1} e^{\left(b_{1}+b_{2} I S\right)\left(E_{2}-E_{1}\right)}$ & 4 \\
$N_{2}=\left(N_{1}^{b_{1}}+\frac{b_{2}}{I S} b_{2}\left(E_{2}^{b_{3}}-E_{1}^{b_{3}}\right)\right)^{1 / b_{1}}$ & 5 \\
$N_{2}=N_{1} e^{b_{1} I S\left(E_{2}-E_{1}\right)}$ & 6 \\
$N_{2}=N_{1} e^{b_{1} I S\left(b_{2}^{E_{2}}-b_{2}^{E_{1}}\right)}$ & 6 \\
\hline
\end{tabular}

Donde: $N_{1}$ y $N_{2}$ es el número de árboles ha-1 observados a la edad $E_{1}$ y $E_{2}, E_{l}$ y $E_{2}$ son la edad de predicción y de proyección, respectivamente; IS es el índice de sitio (m), $e$ representa la función exponencial; y $b_{i}$ son parámetros a estimar.

TABLA 2. Sistemas de crecimiento y rendimiento en área basal y en volumen evaluados.

\begin{tabular}{|c|c|c|c|c|}
\hline Sistema & Modelo de predicción & & Modelo de proyección & \\
\hline S1 & $B_{1}=e^{C_{0}} A_{1}^{C_{1}} e^{C_{2} / E_{1}}$ & 7 & $B_{2}=B_{1} \frac{A_{2}^{C_{1}} e^{-C_{2} / E_{2}}}{A_{1}^{C_{1}} e^{-C_{2} / E_{1}}}$ & 8 \\
\hline S2 & $B_{1}=e^{C_{0}} A_{1}^{C_{1}} e^{\left(C_{2}+C_{3} N_{1}\right) / E_{1}}$ & 9 & $B_{2}=B_{1} \frac{A_{2}^{C_{1}} e^{\left(C_{2}+C_{3} N_{2}\right) / E_{2}}}{A_{1}^{C_{1}} e^{\left(C_{2}+C_{3} N_{1}\right) / E_{1}}}$ & 10 \\
\hline S3 & $B_{1}=e^{C_{0}} A_{1}^{C_{1}} e^{\left(C_{2}+C_{3} N_{1}+C_{4} A_{1}\right) / E_{1}}$ & 11 & $B_{2}=B_{1} \frac{A_{2}^{C_{1}} e^{\left(C_{2}+C_{3} N_{2}+C_{4} A_{2}\right) / E_{2}}}{A_{1}^{C_{1}} e^{\left(C_{2}+C_{3} N_{1}+C_{4} A_{1}\right) / E_{1}}}$ & 12 \\
\hline S4 & $V_{1}=D_{0} B_{1}^{D_{1}} A_{1}^{D_{2}} e^{D_{3} N_{1} / E_{1}}$ & 13 & $V_{2}=V_{1} \frac{B_{2}^{D_{1}} A_{2}^{D_{2}} e^{D_{3} N_{2} / E_{2}}}{B_{1}^{D_{1}} A_{1}^{D_{2}} e^{D_{3} N_{1} / E_{1}}}$ & 14 \\
\hline S5 & $V_{1}=D_{0} B_{1}^{D_{1}} e^{-D_{2} / E_{1}} e^{\left(D_{3} B_{1} / E_{1}+D_{4} A_{1} / E_{1}\right)}$ & 15 & $V_{2}=V_{1} \frac{B_{2}^{D_{1}} e^{-D_{2} / E_{2}} e^{\left(D_{3} B_{2} / E_{2}+D_{4} A_{2} / E_{2}\right)}}{B_{1}^{D_{1}} e^{-D_{2} / E_{1}} e^{\left(D_{3} B_{1} / E_{1}+D_{4} A_{1} / E_{1}\right)}}$ & 16 \\
\hline S6 & $V_{1}=D_{0} B_{1}^{D_{1}} A_{1}^{D_{2}} N_{1}^{D_{3}} e^{\left(D_{4} A_{1} / E_{1}+D_{5} N_{1} / E_{1}\right)}$ & 17 & $V_{2}=V_{1} \frac{B_{2}^{D_{1}} A_{2}^{D_{2}} N_{2}^{D_{3}} e^{\left(D_{4} A_{2} / E_{2}+D_{5} N_{2} / E_{2}\right)}}{B_{1}^{D_{1}} A_{1}^{D_{2}} N_{1}^{D_{3}} e^{\left(D_{4} A_{1} / E_{1}+D_{5} N_{1} / E_{1}\right)}}$ & 18 \\
\hline
\end{tabular}

$B_{1}$ y $B_{2}$ son el área basal a la edad $E_{1}$ y $E_{2} ; A_{1}$ y $A_{2}$ son la altura dominante a la edad $E_{l}$ y $E_{2} ; V_{1}$ y $V_{2}$ son el volumen a la edad $E_{l}$ y $E_{2} ; C_{i}$ y $D_{i}$ son parámetros a estimar, lo demás ya fue definido previamente. 
El ajuste de cada modelo de mortalidad se realizó mediante mínimos cuadrados no lineales (Steel y Torrie, 1997). Cada sistema de crecimiento para área basal y volumen se ajustó de manera simultánea y compatible mediante la técnica de regresión aparentemente no correlacionada (SUR, por sus siglas en inglés) (Zellner, 1962; Rose y Lynch, 2001) con el procedimiento Model de SAS/ETS ${ }^{\circledR}$ (SAS Institute Inc., 2011).

Para el mejor sistema de crecimiento en volumen, se verificó la homocedasticidad mediante inspección gráfica de los residuales en comparación con los predichos; para verificar ausencia de autocorrelación se aplicó la prueba de Durbin-Watson (Steel y Torrie, 1997; Draper y Smith, 1998).

Se seleccionó como el mejor modelo y sistema aquel cuyos parámetros resultaran con la mayor significancia estadística y que mantuviera el más alto balance entre estadísticos de bondad de ajuste aceptables y tendencias biológicas de crecimiento, esto último verificado mediante un análisis visual y comprobación gráfica de que las tendencias de las curvas generadas por cada modelo se sobrepusieran al patrón de las trayectorias observadas (Tamarit et al., 2014b; Hirigoyen, Franco y Diéguez, 2018).

Los criterios de bondad de ajuste fueron el coeficiente de determinación corregido por el número de parámetros ( $\left.\mathrm{R}^{2}{ }_{\text {adj }}\right)$, la raíz del cuadrado medio del error (RCME), el sesgo y el criterio de información de Akaike (AIC) (Parra et al., 2017; Fierros-Mateo et al., 2017). El modelo será mejor cuando presente bajos valores en RCME, sesgo y AIC, además de alto valor en $\mathrm{R}^{2}$ adj. Estos estadísticos fueron calculados como:

$$
R_{a d j}^{2}=1-\left(1-R^{2}\right)\left(\frac{n-1}{n-p}\right)
$$

siendo $R^{2}=1-\frac{\sum_{i=1}^{n}\left(Y_{i}-\hat{Y}_{i}\right)^{2}}{\sum_{i=1}^{n}\left(Y_{i}-\bar{Y}_{i}\right)^{2}}$

$$
\begin{gathered}
\text { RCME }=\sqrt{\frac{\sum_{i=1}^{n}\left(Y_{i}-\hat{Y}_{i}\right)^{2}}{n-p}} \\
\text { Sesgo }=\sum_{i=1}^{n} \frac{Y_{i}-\hat{Y}_{i}}{n}
\end{gathered}
$$

$$
A I C=2 p+n \ln \left(\frac{\sum_{i=1}^{n}\left(Y_{i}-\hat{Y}_{i}\right)^{2}}{n}\right)
$$

Donde $\hat{Y}_{i}, Y_{i}$ y $\bar{Y}_{i}$ son los valores estimados, observados y promedios de la variable dasométrica en cuestión, respectivamente; $n$ es el número total de observaciones usadas en el ajuste de los modelos; $p$ es el número de parámetros del modelo.

Después de seleccionar el mejor sistema de crecimiento para área basal y para volumen de la plantación completa, en cada sistema se evaluó el efecto de la densidad de plantación inicial en el crecimiento mediante la incorporación de variables indicadoras (Steel y Torrie, 1997), lo que permitió determinar si es o no suficiente disponer de un solo sistema para ambas densidades. Posteriormente, y con un propósito similar, en ambos sistemas de crecimiento y para cada densidad de plantación inicial se evaluó el efecto del sitio de plantación (I, II, III y IV) en el crecimiento, también usando variables indicadoras.

Con base en Castedo, Barrio, Parresol y Álvarez (2005), cada parámetro de los modelos del sistema se expandió al incluirle un parámetro asociado con su correspondiente variable indicadora, tomando la forma general $\beta_{i}+b_{i} I$, donde $\beta_{i}$ representa cada parámetro del modelo reducido en el sistema, $b_{i}$ es el parámetro asociado al modelo completo e $I$ es la variable indicadora que toma el valor de 0 o 1 . De esta manera, la densidad de plantación inicial y el sitio de plantación como variables cualitativas con $t$ niveles, fueron consideradas con $t-1$ variables indicadoras.

Con los modelos dinámicos de crecimiento seleccionados para cada variable de totalidad de rodal a escala de la plantación completa, a escala de densidad de plantación inicial y a escala de sitio de plantación, se realizó una proyección en la que se calculó el valor de cada variable a diferentes edades y hasta el turno planeado a 19 años. Adicionalmente, se construyeron curvas de crecimiento del volumen total por hectárea con corteza para las calidades de índice sitio baja, media y alta (12 m, $15 \mathrm{~m}$ y $18 \mathrm{~m})$ con una edad de referencia de 7.5 años. 


\section{Resultados}

\section{Selección del modelo de mortalidad}

Para la densidad de $1250 \mathrm{pl} \mathrm{ha} \mathrm{p}^{-1}$, aun cuando los modelos de mortalidad 2 y 4 presentaron en conjunto los mejores valores en los criterios de bondad de ajuste (Tabla 3), se determinó que las curvas de mortalidad promedio no se sobreponen a la tendencia de mortalidad observada. El modelo 3 presentó deficientes estadísticos de ajuste y proyectó valores incongruentes de densidad, motivo por el cual fue descartado. En cambio, el modelo 5, comparativamente presentó buenos estadísticos de ajuste, sobre todo en el sesgo y la precisión; ambos expresados en las unidades de la variable analizada, el primero es indicativo de que se tiene la mínima desviación del modelo con respecto a los valores observados, el segundo, dado por el menor valor de la RCME, da idea del error medio que comete el modelo. La tendencia de la curva que genera siguió de mejor manera el patrón observado, por lo que se seleccionó para estimar la reducción del número de árboles por hectárea en el tiempo.

TABLA 3. Parámetros estimados y estadísticos de ajuste de los modelos de mortalidad evaluados por densidad de plantación inicial.

\begin{tabular}{|c|c|c|c|c|c|c|c|c|c|}
\hline Densidad & Modelo & $\begin{array}{l}R C M E \\
\left(N h a^{-1}\right)\end{array}$ & $\begin{array}{l}\text { Sesgo } \\
\left(N h a^{-1}\right)\end{array}$ & $R^{2} a d j$ & $A / C$ & Parámetro & $\begin{array}{c}\text { Valor } \\
\text { estimado }\end{array}$ & E.E. & $\operatorname{Pr}>|t|$ \\
\hline \multirow{11}{*}{$\begin{array}{l}1250 \\
\mathrm{pl} \mathrm{ha}^{-1}\end{array}$} & 1 & 124.8001 & 6.5758 & 0.7233 & 12415.31 & $b_{1}$ & -0.01178 & 0.00039 & $<0.0001$ \\
\hline & 2 & 112.9333 & -6.2853 & 0.7735 & 12158.32 & $b_{1}$ & -4.64373 & 0.00433 & $<0.0001$ \\
\hline & & & & & & $\mathrm{b}_{2}{ }^{*}$ & 8.96E-16 & - & - \\
\hline & 3 & 124.4228 & 6.3803 & 0.7250 & 12408.52 & $b_{1}$ & -0.00266 & 0.00204 & $<0.0001$ \\
\hline & & & & & & $b_{2}$ & -0.00070 & 0.00014 & $<0.0001$ \\
\hline & 4 & 110.6488 & -13.5445 & 0.7825 & 12107.76 & $b_{1}$ & -12.0673 & 1.28900 & $<0.0001$ \\
\hline & & & & & & $b_{2}$ & $7.83 E-42$ & 7.91E-41 & $<0.0001$ \\
\hline & & & & & & bз & 4.073067 & 0.39020 & $<0.0001$ \\
\hline & 5 & 124.4101 & 6.1007 & 0.7251 & 12407.26 & $b_{1}$ & -0.00089 & 0.00002 & $<0.0001$ \\
\hline & 6 & 121.4420 & 5.5050 & 0.7380 & 12346.15 & $b_{1}$ & 0.981012 & 0.00268 & $<0.0001$ \\
\hline & & & & & & $b_{2}$ & 0.108135 & 0.00600 & $<0.0001$ \\
\hline \multirow{11}{*}{$\begin{array}{c}816 \\
\mathrm{pl} \mathrm{ha}^{-1}\end{array}$} & 1 & 68.7812 & -5.3107 & 0.7619 & 2251.85 & $b_{1}$ & -0.00606 & 0.00059 & $<0.0001$ \\
\hline & 2 & 72.6715 & -15.6720 & 0.7342 & 2281.12 & $b_{1}{ }^{*}$ & $8.96 \mathrm{E}-16$ & - & \\
\hline & & & & & & $b_{2}$ & $2.36 \mathrm{E}-17$ & 0.02030 & $<0.0001$ \\
\hline & 3 & 63.9327 & -3.8836 & 0.7943 & 2213.96 & $b_{1}$ & -0.02208 & 0.00338 & $<0.0001$ \\
\hline & & & & & & $b_{2}$ & 0.001297 & 0.00030 & $<0.0001$ \\
\hline & 4 & 65.1683 & -2.0679 & 0.7870 & 2225.13 & $b_{1}$ & $-5.04 E-07$ & $1.33 E-6$ & $<0.0001$ \\
\hline & & & & & & $b_{2}$ & $9.84 \mathrm{E}-07$ & 0.00002 & $<0.0001$ \\
\hline & & & & & & $\mathrm{b}_{3}$ & 0.32350 & 0.43380 & $<0.0001$ \\
\hline & 5 & 72.6705 & -12.5667 & 0.7342 & 2225.13 & $b_{1}$ & -0.00041 & 0.00005 & $<0.0001$ \\
\hline & 6 & 72.7442 & -12.6776 & 0.7336 & 2282.65 & $b_{1}$ & 0.988311 & 0.02970 & $<0.0001$ \\
\hline & & & & & & $b_{2}$ & 0.046581 & 0.08970 & $<0.0001$ \\
\hline
\end{tabular}

RCME = raíz del cuadrado medio del error, $R^{2}$ adj = coeficiente de determinación corregido por el número de parámetros, AlC = criterio de información de $A$ kaike, pl ha-1 = plantas por hectárea, E.E. = error estándar. *el resultado del ajuste mejoró cuando el valor de este parámetro se mantuvo fijo. 
El modelo 5, si bien no presentó el mejor valor en el AIC, explicó $72 \%$ de la variabilidad total presente y es parsimonioso, ya que solo tiene un parámetro significativo que está relacionado para considerar el efecto del IS de manera directamente proporcional; característica relevante que permite comparar el declive de la densidad entre diferentes calidades de estación. El segundo mejor modelo que describe la tendencia referida fue el 6 , cuya tasa relativa de cambio en el número de árboles es proporcional a la función exponencial de la edad, resultando con alta significancia en sus dos parámetros y también considera el efecto del IS. Por su parte, el modelo 1, aun cuando presentó los valores más bajos en los criterios de ajuste, la curva promedio de mortalidad que describe se apega a los datos observados, razón por la que puede considerarse como modelo confiable y robusto a utilizarse cuando no se conoce el IS.

Para la densidad de $816 \mathrm{pl} \mathrm{ha-1}$, los modelos presentaron comportamientos similares a los antes referidos, los modelos con el mejor ajuste estadístico fueron el 5 y el 6, aunque el sesgo fue ligeramente mayor, lo cual se debió al menor número de observaciones y a que el intervalo de edades fue más corto. El modelo 1, aun con el inconveniente señalado, puede considerarse alternativo. Los modelos que tienen valores negativos en sus parámetros tienden a sobreestimar la variable, sucede lo contrario con los modelos con signo positivo.

\section{Selección del sistema de crecimiento para área basal}

Para los sistemas de crecimiento en área basal se determinó que, aunque comparativamente en todos los casos los parámetros resultaron confiables, los estadísticos muestran que el sistema S3 presenta la mayor precisión (menor valor en la RCME) y el menor sesgo (Tabla 4), ambos expresados en las unidades de la variable analizada; además, su modelo de proyección 12 presentó el más alto valor en el $\mathrm{R}^{2}$ adj y el menor valor en el AIC, lo que evidencia mayor verosimilitud y garantiza proyecciones más confiables de área basal a determinada edad. Bajo el mismo contexto, el segundo mejor sistema fue el S2. Una comparación de los modelos de predicción de los tres sistemas al usar el IS promedio de $15 \mathrm{~m}$ (edad de referencia de 7.5 años) para la plantación de teca en general, mostró que los modelos 9 y 11 de los sistemas S2 y S3, respectivamente reprodujeron un patrón muy similar en el intervalo de edad observado; fuera de este, el modelo 11 estimó valores de área basal, comparativamente mayores, que van en concordancia con las tendencias crecientes observadas, razón por la que se consideró que el sistema S3 es el más apropiado para predecir y proyectar el crecimiento en área basal de las plantaciones de teca en la zona de estudio.

\section{Selección del sistema de crecimiento para volumen}

En el ajuste simultáneo de los sistemas de crecimiento en volumen, todos los parámetros fueron significativos y sus estadísticos de ajuste similares (Tabla 5), en términos de precisión y sesgo, los sistemas S4 y S6 fueron ligeramente mejores, pues sus valores expresados en las mismas unidades de la variable analizada son menores. Una comparación gráfica de los modelos de predicción de los tres sistemas usando el índice de sitio (IS) promedio de 15 m (Fig. 1) revela que, en el intervalo de edad observado, los tres modelos de predicción describen un patrón similar entre ellos, apegado a la condición promedio observada. Sin embargo, fuera de ese intervalo, los modelos de predicción 13 y 17 de los sistemas S4 y S6, respectivamente, presentan patrones más consistentes con respecto a las trayectorias crecientes observadas. Al comparar ambos sistemas, se seleccionó al S4 como el mejor, tanto para predecir como para proyectar el crecimiento en volumen de plantaciones de teca en general, tiene la ventaja adicional de ser parsimonioso en cuatro parámetros. El sistema S5 realizó predicciones comparativamente inferiores y más conservadoras, por lo que se descartó para describir el crecimiento en volumen. 
TABLA 4. Parámetros estimados y estadísticos de ajuste de los modelos que componen cada sistema de crecimiento evaluados para el área basal.

\begin{tabular}{cccccccccc}
\hline Sistema & Modelo & $\begin{array}{c}R C M E \\
\left(m^{2} h a^{-1}\right)\end{array}$ & $\begin{array}{c}\text { Sesgo } \\
\left(m^{2} h a^{-1}\right)\end{array}$ & $R^{2}$ adj & AIC & Parámetro & $\begin{array}{c}\text { Valor } \\
\text { estimado }\end{array}$ & $\begin{array}{c}\text { Error } \\
\text { estándar }\end{array}$ & Pr>|t| \\
\hline S1 & 7 & 1.5718 & -0.4776 & 0.8037 & 2835.4 & $C_{0}$ & 0.313606 & 0.0660 & $<0.0001$ \\
& 8 & 1.1151 & 0.2974 & 0.9208 & 1428.2 & $C_{1}$ & 1.031216 & 0.0224 & $<0.0001$ \\
& & & & & & $C_{2}$ & 6.069035 & 0.7327 & $<0.0001$ \\
\hline S2 & 9 & 1.2047 & -0.3498 & 0.8847 & 1667.5 & $C_{0}$ & -0.47840 & 0.0555 & $<0.0001$ \\
& 10 & 0.9176 & 0.1447 & 0.9464 & 968.3 & $C_{1}$ & 1.186046 & 0.0193 & $<0.0001$ \\
& & & & & & $C_{2}$ & -36.2516 & 1.1004 & $<0.0001$ \\
& & & & & & $C_{3}$ & 0.027658 & 0.0008 & $<0.0001$ \\
\hline S3 & 11 & 1.2150 & -0.3338 & 0.8827 & 1696.3 & $C_{0}$ & -0.65399 & 0.0738 & $<0.0001$ \\
& 12 & 0.9099 & 0.1231 & 0.9472 & 952.9 & $C_{1}$ & 1.278159 & 0.0314 & $<0.0001$ \\
& & & & & & $C_{2}$ & -33.6374 & 1.3842 & $<0.0001$ \\
& & & & & & $C_{3}$ & 0.028354 & 0.0008 & $<0.0001$ \\
& & & & & & $C_{4}$ & -0.51966 & 0.1409 & 0.0002 \\
\hline
\end{tabular}

$R C M E$ = raíz del cuadrado medio del error, $R^{2}$ adj = coeficiente de determinación corregido por el número de parámetros, AIC = criterio de información de $A$ kaike.

TABLA 5. Parámetros estimados y estadísticos de ajuste de los modelos que componen cada sistema de crecimiento evaluados para el volumen.

\begin{tabular}{|c|c|c|c|c|c|c|c|c|c|}
\hline Sistema & Modelo & $\begin{array}{l}\text { RCME } \\
\left(m^{2} h a^{-1}\right)\end{array}$ & $\begin{array}{c}\text { Sesgo } \\
\left(m^{3} h a^{-1}\right)\end{array}$ & $R^{2}$ adj & $A I C$ & Parámetro & $\begin{array}{c}\text { Valor } \\
\text { estimado }\end{array}$ & $\begin{array}{c}\text { Error } \\
\text { estándar }\end{array}$ & $\operatorname{Pr}>|t|$ \\
\hline \multirow[t]{4}{*}{$\mathrm{S} 4$} & 13 & 1.0333 & -0.0850 & 0.9971 & 1228.0 & Do & 0.943286 & 0.01930 & $<0.0001$ \\
\hline & 14 & 1.2548 & -0.1962 & 0.9971 & 1808.1 & $D_{1}$ & 1.034274 & 0.00519 & $<0.0001$ \\
\hline & & & & & & $\mathrm{D}_{2}$ & 0.636922 & 0.01120 & $<0.0001$ \\
\hline & & & & & & $\mathrm{D}_{3}$ & 0.000251 & 0.00013 & 0.0445 \\
\hline \multirow[t]{5}{*}{ S5 } & 15 & 1.0943 & -0.0694 & 0.9968 & 1377.2 & Do & 2.085816 & 0.03310 & $<0.0001$ \\
\hline & 16 & 1.4213 & -0.1890 & 0.9962 & 2319.1 & $D_{1}$ & 1.371174 & 0.00720 & $<0.0001$ \\
\hline & & & & & & $\mathrm{D}_{2}$ & 9.766170 & 0.35020 & $<0.0001$ \\
\hline & & & & & & $\mathrm{D}_{3}$ & -1.82320 & 0.05240 & $<0.0001$ \\
\hline & & & & & & $D_{4}$ & 2.471863 & 0.04340 & $<0.0001$ \\
\hline \multirow[t]{6}{*}{56} & 17 & 0.8635 & -0.0718 & 0.9980 & 860.4 & Do & 1.512010 & 0.06890 & $<0.0001$ \\
\hline & 18 & 1.1656 & -0.0988 & 0.9975 & 1561.2 & $D_{1}$ & 1.092857 & 0.00561 & $<0.0001$ \\
\hline & & & & & & $D_{2}$ & 0.471515 & 0.01230 & $<0.0001$ \\
\hline & & & & & & $D_{3}$ & -0.044420 & 0.00583 & $<0.0001$ \\
\hline & & & & & & $D_{4}$ & 0.613020 & 0.02480 & $<0.0001$ \\
\hline & & & & & & $D_{5}$ & -0.002390 & 0.00020 & $<0.0001$ \\
\hline
\end{tabular}

$R C M E$ = raíz del cuadrado medio del error, $R^{2}$ adj = coeficiente de determinación corregido por el número de parámetros, AIC = criterio de información de $A$ kaike . 


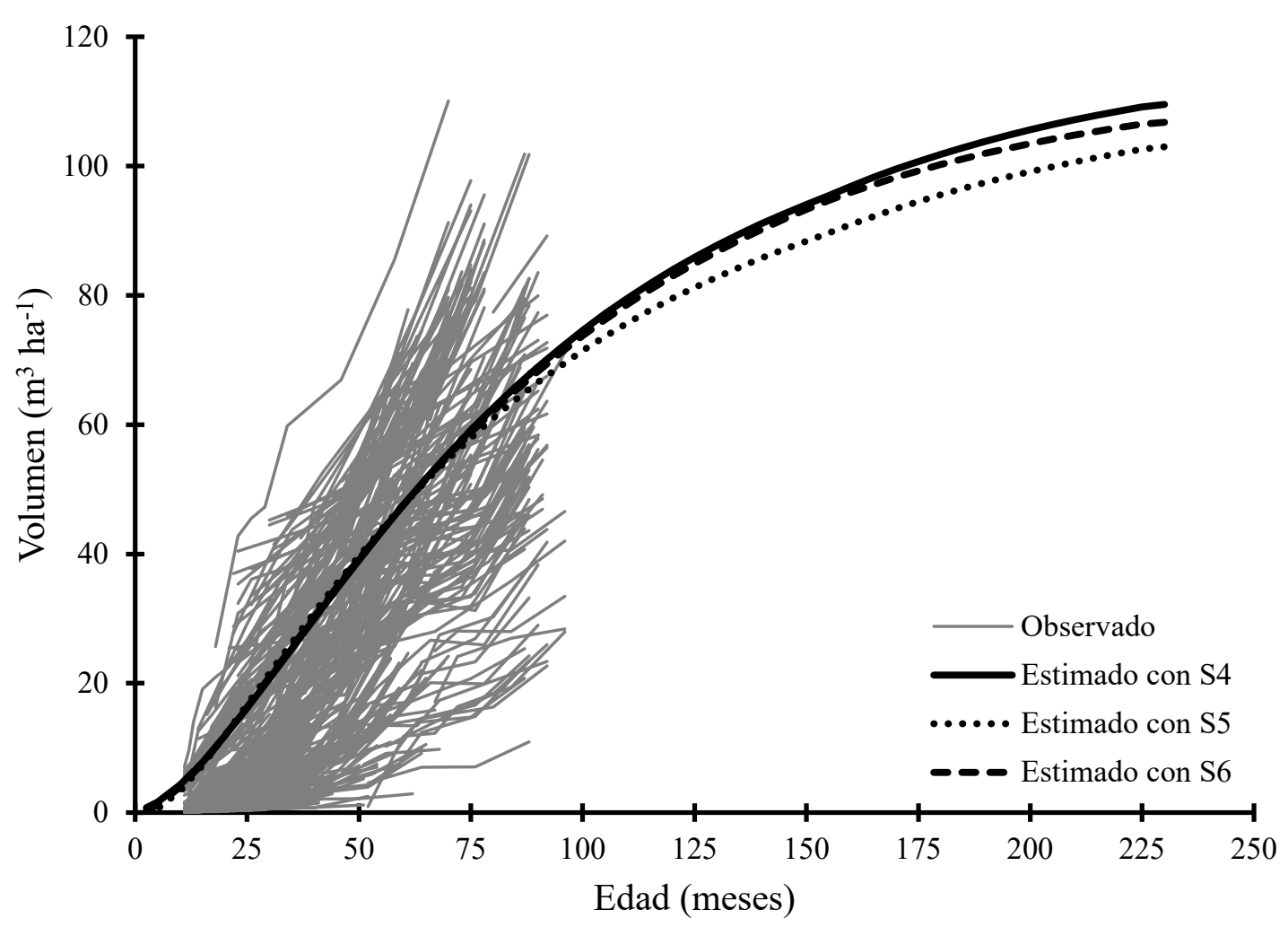

FIGURA 1. Comparativo del crecimiento en volumen estimado con los modelos de predicción de los sistemas evaluados para el IS promedio de $15 \mathrm{~m}$ en la PFC de teca.

El comportamiento gráfico de los residuales del volumen con respecto a los predichos del volumen que proporciona el sistema S4 (Fig. 2) evidencian un patrón tendiente a ser homocedástico (Steel y Torrie, 1997). El valor de la prueba de Durbin-Watson fue de 2.2065 para el modelo de proyección 14, el cual es próximo al valor teórico de 2.0, lo que indica ausencia de autocorrelación (Draper y Smith, 1998).

\section{Crecimiento en área basal por densidad inicial y sitio de plantación}

La incorporación de variables indicadoras en los modelos 11 y 12 del sistema S3 para el área basal detectó un efecto significativo que sugiere que en cada densidad se deben tener valores diferentes en sus parámetros. Debido a que el comportamiento gráfico evidenció inconsistencias y comportamientos biológicos poco probables, se decidió realizar el ajuste por separado para cada tipo de densidad (Tabla 6). El mejor ajuste y comportamiento gráfico de las tendencias estimadas con el modelo de predicción 11 se obtuvo cuando el parámetro $\mathrm{C}_{0}$ se utilizó sin ser afectado por la función exponencial.

El efecto del sitio de plantación sobre el crecimiento en área basal se presenta solo para la densidad de plantación inicial de $1250 \mathrm{pl} \mathrm{ha}{ }^{-1}$ incorporando variables indicadoras para el ajuste del sistema S3 (Tabla 7), para la densidad de $816 \mathrm{pl} \mathrm{ha}^{-1}$ los ajustes fueron inconsistentes debido al reducido número de observaciones y al estrecho intervalo de edad observado, razón por la que se omite su presentación. Se determinó que no es posible usar un sistema de crecimiento en área basal único para todos los sitios, ya que se detectaron diferencias estadísticas significativas en el crecimiento de los sitios I, II y IV. Aun cuando los parámetros del sistema en los sitios I y III son 


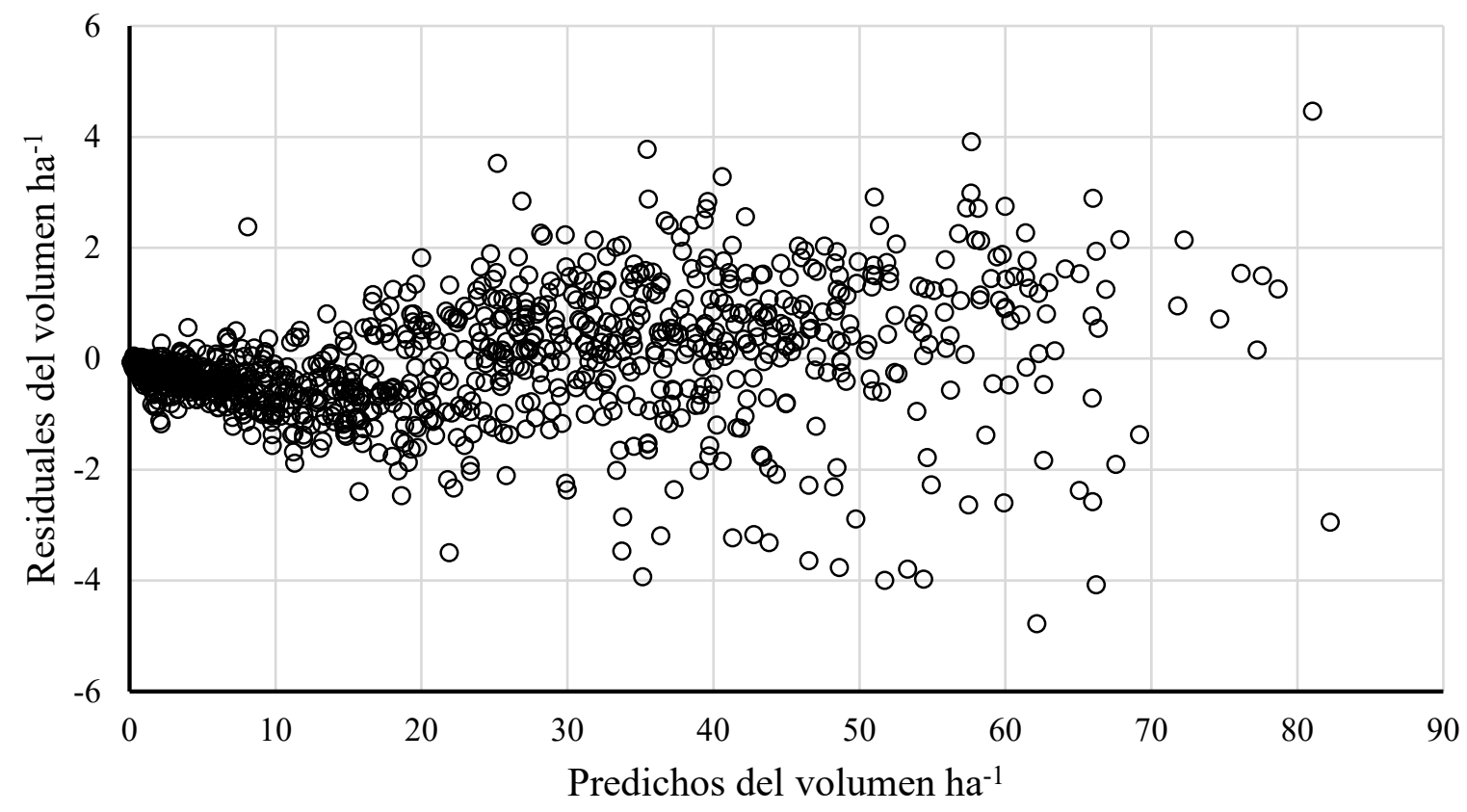

FIGURA 2. Comportamiento gráfico de los residuales frente a predichos del volumen por hectárea que proporciona el sistema de crecimiento S4.

TABLA 6. Parámetros estimados y estadísticos de ajuste del sistema S3 de crecimiento en área basal considerando el tipo de densidad de plantación inicial.

\begin{tabular}{|c|c|c|c|c|c|c|c|c|}
\hline $\begin{array}{c}\text { Densidad } \\
\text { inicial }\end{array}$ & Modelo & $\begin{array}{c}\text { RECM } \\
\left(m^{2} h a^{-1}\right)\end{array}$ & $\begin{array}{c}\text { Sesgo } \\
\left(m^{2} h a^{-1}\right)\end{array}$ & $R^{2} a d j$ & Parámetro & $\begin{array}{c}\text { Valor } \\
\text { estimado }\end{array}$ & $\begin{array}{c}\text { Error } \\
\text { estándar }\end{array}$ & $\operatorname{Pr}>|t|$ \\
\hline 1250 & 11 & 1.2599 & -0.2904 & 0.8542 & $C_{0}$ & 0.910589 & 0.0859 & $<0.0001$ \\
\hline \multirow[t]{4}{*}{$\mathrm{pl} \mathrm{ha}^{-1}$} & 12 & 0.9568 & 0.1556 & 0.9472 & $\mathrm{C}_{1}$ & 1.039485 & 0.0391 & $<0.0001$ \\
\hline & & & & & $\mathrm{C}_{2}$ & -51.0304 & 2.3754 & $<0.0001$ \\
\hline & & & & & $\mathrm{C}_{3}$ & 0.032679 & 0.0011 & $<0.0001$ \\
\hline & & & & & $\mathrm{C}_{4}$ & 0.909013 & 0.1889 & $<0.0001$ \\
\hline 816 & 11 & 0.3783 & -0.0585 & 0.7138 & Co & 0.251630 & 0.0446 & $<0.0001$ \\
\hline \multirow[t]{4}{*}{$\mathrm{pl} \mathrm{ha}^{-1}$} & 12 & 0.5586 & 0.1035 & 0.8622 & $\mathrm{C}_{1}$ & 1.513149 & 0.0933 & $<0.0001$ \\
\hline & & & & & $C_{2}$ & -53.0825 & 4.6805 & $<0.0001$ \\
\hline & & & & & $C_{3}$ & 0.066297 & 0.0050 & $<0.0001$ \\
\hline & & & & & $\mathrm{C}_{4}$ & -2.10949 & 0.3789 & $<0.0001$ \\
\hline
\end{tabular}

$R C M E$ = raíz del cuadrado medio del error, $R^{2}$ adj = coeficiente de determinación corregido por el número de parámetros, pl ha-1 = plantas por hectárea 
TABLA 7. Parámetros y estadísticos de ajuste del sistema S3 del crecimiento en área basal por sitio de plantación para la densidad de $1250 \mathrm{pl} \mathrm{ha}^{-1}$.

\begin{tabular}{|c|c|c|c|c|c|c|c|}
\hline Modelo & $\begin{array}{c}R C M E \\
\left(m^{2} h a^{-1}\right)\end{array}$ & $\begin{array}{c}\text { Sesgo } \\
\left(m^{2} h a^{-1}\right)\end{array}$ & $R^{2}$ adj & Parámetro & $\begin{array}{c}\text { Valor } \\
\text { estimado* }\end{array}$ & $\begin{array}{c}\text { Error } \\
\text { estándar }\end{array}$ & $\operatorname{Pr}>|t|$ \\
\hline 11 & 1.0513 & -0.1691 & 0.9398 & Coo & -0.79756 & 0.0990 & $<0.0001$ \\
\hline \multirow[t]{8}{*}{12} & 0.9126 & 0.0433 & 0.8985 & $\mathrm{COl}$ & -0.46094 & 0.0883 & $<0.0001$ \\
\hline & & & & $\mathrm{CO} 3$ & 1.01342 & 0.0987 & $<0.0001$ \\
\hline & & & & $\mathrm{C}_{20}$ & 1.27542 & 0.0395 & $<0.0001$ \\
\hline & & & & $\mathrm{C}_{21}$ & 0.197731 & 0.0355 & $<0.0001$ \\
\hline & & & & $c_{23}$ & -0.56452 & 0.0440 & $<0.0001$ \\
\hline & & & & $C_{3}$ & -48.24700 & 2.1575 & $<0.0001$ \\
\hline & & & & $\mathrm{C}_{4}$ & 0.03151 & 0.0010 & $<0.0001$ \\
\hline & & & & $\mathrm{C}_{5}$ & 1.37102 & 0.1690 & $<0.0001$ \\
\hline
\end{tabular}

RCME = raíz del cuadrado medio del error, $R^{2}$ adi = coeficiente de determinación corregido por el número de parámetros. *El parámetro Co para el sitio I y III es de -0.79756,

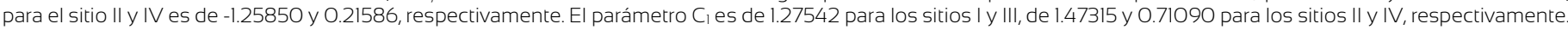

iguales, el crecimiento en área basal entre estos sitios se ve afectado debido a que los parámetros de la ecuación dinámica de A e IS, que es una variable explicativa en el sistema S3, toman valores diferentes y en consecuencia, el crecimiento en área basal es ligeramente mayor en el sitio II.

\section{Crecimiento en volumen por densidad inicial y sitio de plantación}

La incorporación de variables indicadoras en los modelos 13 y 14 del sistema S4 de crecimiento en volumen para evaluar el efecto de la densidad de plantación inicial detectó una diferencia estadística significativa en el parámetro asintótico (Tabla 8); para la densidad de $1250 \mathrm{pl} \mathrm{ha}^{-1}$ el parámetro $\mathrm{D}_{0}$ toma el valor de 0.941381 y para la densidad de $816 \mathrm{pl} \mathrm{ha}^{-1}$ de 0.866177 .

El efecto del sitio de plantación sobre el crecimiento en volumen se presenta solo para la densidad de plantación inicial de $1250 \mathrm{pl} \mathrm{ha}^{-1}$ incorporando variables indicadoras para ajustar el sistema S4; para la densidad de $816 \mathrm{pl} \mathrm{ha}^{-1}$ los ajustes no se presentan debido a que fueron inconsistentes por las mismas razones ya referidas para el área basal. Se detectó una diferencia significativa en el parámetro $\mathrm{D}_{3}$, lo que evidencia que existen diferentes tasas de crecimiento entre los cuatro sitios de plantación evaluados (Tabla 9).
Los sitios I y II son diferentes del resto siendo $\mathrm{D}_{3}=$ 0.000309 , en tanto que para los sitios III y IV toman el valor de -0.000081 y -0.001071 , respectivamente.

\section{DISCUSIÓN}

\section{Modelo de mortalidad}

La tasa de mortalidad que describe el modelo 5, para un determinado índice de sitio, fue comparativamente mayor para la más alta densidad de plantación después de los 50 meses de edad. Este modelo sugiere que la mortalidad aumenta ligeramente al incrementar el IS, resultado que coincide con el patrón de mortalidad observado por Álvarez-González, Castedo-Dorado, Ruiz-González, López-Sánchez y Gadow (2004) para rodales naturales de Pinus radiata en España, al determinado por DiéguezAranda et al. (2005) y Zhao et al. (2007) para plantaciones de Pinus sylvestris en España y P. taeda en Estados Unidos, respectivamente, quienes señalan que la mortalidad por competencia se presenta a edades tempranas en las mejores calidad de sitio, agregan que esta tendencia puede parcialmente atribuirse a la mayor competencia local que se presenta en las mejores calidades de estación y a la disponibilidad de nutrimentos claves (nitrógeno, fósforo, potasio y magnesio), así como a las diferencias en la capacidad de infiltración del suelo. 
TABLA 8. Parámetros estimados y estadísticos de ajuste del sistema S4 de crecimiento en volumen considerando el tipo de densidad de plantación inicial.

\begin{tabular}{cccccccc}
\hline Modelo & $\begin{array}{c}\text { RECM } \\
\left(\mathrm{m}^{3} \mathrm{ha}^{-1}\right)\end{array}$ & $\begin{array}{c}\text { Sesgo } \\
\left(\mathrm{m}^{3} \mathrm{ha} \mathrm{a}^{-1}\right)\end{array}$ & $R^{2}{ }_{\text {adj }}$ & Parámetro & $\begin{array}{c}\text { Valor } \\
\text { estimado }\end{array}$ & $\begin{array}{c}\text { Error } \\
\text { estándar }\end{array}$ & Pr ||$t \mid$ \\
\hline$(13)$ & 1.0297 & -0.0527 & 0.9971 & $\mathrm{D}_{00}$ & 0.866177 & 0.02310 & $<0.0001$ \\
$(14)$ & 1.2525 & -0.1844 & 0.9971 & $\mathrm{~d}_{1}$ & 0.075204 & 0.01480 & $<0.0001$ \\
& & & $\mathrm{D}_{1}$ & 1.030964 & 0.00521 & $<0.0001$ \\
& & & $\mathrm{D}_{2}$ & 0.640692 & 0.01120 & $<0.0001$ \\
& & & $\mathrm{D}_{3}$ & 0.000275 & 0.00012 & 0.0274 \\
\hline
\end{tabular}

$\mathrm{RCME}$ = raíz del cuadrado medio del error, $\mathrm{R}^{2}$ adj = coeficiente de determinación corregido por el número de parámetros.

TABLA 9. Parámetros estimados y estadísticos del ajuste del sistema S4 de crecimiento en volumen considerando el efecto del sitio de plantación cuando $\mathrm{N}=1250 \mathrm{pl} \mathrm{ha} \mathrm{h}^{-1}$.

\begin{tabular}{cccccccc}
\hline Modelo & $\begin{array}{c}R C M E \\
\left(\mathrm{~m}^{3} \mathrm{ha}^{-1}\right)\end{array}$ & $\begin{array}{c}\text { Sesgo } \\
\left(\mathrm{m}^{3} \mathrm{ha}^{-1}\right)\end{array}$ & $R^{2}$ adj & Parámetro & $\begin{array}{c}\text { Valor } \\
\text { estimado }\end{array}$ & $\begin{array}{c}\text { Error } \\
\text { estándar }\end{array}$ & Pr $>|t|$ \\
\hline$(13)$ & 1.1522 & -0.0374 & 0.9962 & $\mathrm{D}_{0}$ & 0.967307 & 0.022700 & $<0.0001$ \\
$(14)$ & 1.3731 & -0.2613 & 0.9963 & $\mathrm{D}_{1}$ & 1.034495 & 0.005950 & $<0.0001$ \\
& & & & $\mathrm{D}_{2}$ & 0.627286 & 0.012800 & $<0.0001$ \\
& & & $\mathrm{D}_{30}$ & 0.000309 & 0.000145 & 0.0331 \\
& & & $\mathrm{~d}_{32}$ & -0.000390 & 0.000137 & 0.0047 \\
& & & $\mathrm{~d}_{33}$ & -0.001380 & 0.000385 & 0.0003 \\
\hline
\end{tabular}

\section{Crecimiento y rendimiento en área basal}

Considerando el límite superior de edad observado de 8 años y el IS promedio de $15 \mathrm{~m}$ (edad de referencia de 7.5 años), el modelo de predicción 11 del sistema S3 para área basal estimó $12.3 \mathrm{~m}^{2} \mathrm{ha}^{-1}$, la cual comparativamente fue inferior a la referida por Bermejo, Cañellas y San Miguel (2004), quienes usando un IS promedio de $21 \mathrm{~m}$ (edad de referencia de 10 años) que es equivalente en incremento medio al IS de $15 \mathrm{~m}$ usado para la zona de estudio, predijeron $16.9 \mathrm{~m}^{2} \mathrm{ha}^{-1}$ a los 8 años en plantaciones de teca en la costa pacífica de Costa Rica. También fue inferior a la estimada por Pereira et al. (2008) en plantaciones de teca con 8 años de edad en la región de Mato Grosso, Brasil, quienes con un IS de $10.5 \mathrm{~m}$ (edad de referencia de 4 años) estimaron $30.5 \mathrm{~m}^{2} \mathrm{ha}^{-1}$, aunque el modelo que usaron no contiene de manera implícita una función de mortalidad.
El área basal proyectada para el IS promedio fuera del intervalo de edad observado y próximo al turno proyectado en 19 años es de $17 \mathrm{~m}^{2} \mathrm{ha}^{-1}$, la cual, aunque es biológicamente razonable, se considera conservadora de acuerdo con las trayectorias y tendencias observadas, misma que puede rectificarse al incorporar nuevas observaciones con edades mayores conforme evolucione la plantación con el tiempo. Comparativamente, para las localidades y condiciones de índice de sitio previamente indicadas, tal estimación es $2.3 \%$ inferior a la registrada por Bermejo et al. (2004) de $17.4 \mathrm{~m}^{2} \mathrm{ha}^{-1}$ a los 20 años de edad de la plantación. Estas diferencias pueden atribuirse, en parte, a las diferentes condiciones de crecimiento, producto de la combinación de varios factores biofísicos, ambientales $\mathrm{y}$ a diferencias en los tratamientos silvícolas empleados. 
Con respecto al crecimiento en área basal por densidad de plantación, se determinó que la densidad de $1250 \mathrm{pl} \mathrm{ha}^{-1}$ presentó comparativamente un mayor crecimiento en área

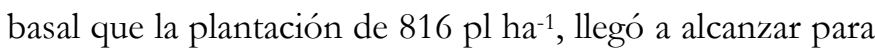
la mejor calidad de sitio (IS $=18 \mathrm{~m}$ ) un estimado de $19 \mathrm{~m}^{2}$ $\mathrm{ha}^{-1}$ contra $17.1 \mathrm{~m}^{2} \mathrm{ha}^{-1}$ a la edad del turno planeado a 19 años, respectivamente, pero sin alcanzar los $21.1 \mathrm{~m}^{2} \mathrm{ha}^{-1}$ proyectados a nivel de toda la plantación en general.

El mayor crecimiento en área basal entre sitios de plantación (para $\mathrm{N}=1250 \mathrm{pl} \mathrm{ha}^{-1}$ ) lo obtuvo el sitio II, el cual, a la edad de rotación de 19 años alcanza $25.8 \mathrm{~m}^{2} \mathrm{ha}^{-1}$ en la mejor calidad de sitio ( $I S=18 \mathrm{~m}$ ), seguido de los sitios I y III con $22.2 \mathrm{~m}^{2} \mathrm{ha}^{-1}$ y $20.9 \mathrm{~m}^{2} \mathrm{ha}^{-1}$, respectivamente. En el sitio IV se considera que se subestima el crecimiento, debido en parte a que las observaciones trabajadas se localizan en un reducido intervalo de edad.

\section{Crecimiento y rendimiento en volumen}

La estimación del rendimiento en volumen de la plantación completa de teca con los modelos 13 y 14 del sistema $\$ 4$ y con el IS promedio de $15 \mathrm{~m}$, fue de $71.8 \mathrm{~m}^{3} \mathrm{ha}^{-1}$ a la edad de 8 años. A la misma edad y con un IS equivalente al usado en este estudio, el rendimiento fue $6.5 \%$ inferior a lo señalado por Bermejo et al. (2004) de $76.8 \mathrm{~m}^{3} \mathrm{ha}^{-1}$ en plantaciones de teca de la costa del Pacífico de Costa Rica. También es comparativamente inferior al observado por Restrepo, Orrego, del Valle y Salazar (2012) para teca establecida en la región noroccidental de Colombia, quienes a una edad de 9.5 años cuantificaron $153.4 \mathrm{~m}^{3} \mathrm{ha}^{-1}$.

En general, el crecimiento y rendimiento promedio en la zona de estudio a la edad de la cosecha proyectada a 19 años, es inferior a la registrada en otros países en los que teca se ha establecido (Nunifu y Murchison, 1999; Pérez y Kanninen 2005; Sunanda y Jayaraman, 2006).

Las diferencias pueden atribuirse a que las prácticas de manejo y regímenes silviculturales que se aplican son diferentes. Sobre todo, es claro que las condiciones climáticas, edáficas, orográficas y topográficas en que se han establecido las plantaciones difieren, particularmente en los intervalos de precipitación y temperatura, así como las características físicas y químicas del suelo, que al combinarse dan lugar a diferentes escenarios de calidad de estación (Álvarez-González et al., 2004; Diéguez-Aranda et al., 2005; Zhao et al., 2007).

Bermejo et al. (2004) indican que las tasas de crecimiento más altas de teca se expresan cuando se ha realizado la correcta y óptima selección del sitio, cumpliendo cabalmente con todos los requerimientos ecológicos para su establecimiento. En contraparte, Pérez y Kanninen (2005) refieren que en Latinoamérica existen plantaciones de teca que no han alcanzado las expectativas de productividad, y que ello se debe a la inapropiada selección del sitio de plantación, al uso de pobre material genético y a prácticas silviculturales incorrectas.

El efecto de la densidad de plantación inicial se refleja en diferencias del crecimiento en volumen, en la edad de rotación planeada a 19 años, en la mejor calidad de sitio (18 m) se estiman $136.8 \mathrm{~m}^{3} \mathrm{ha}^{-1}$ y $113.1 \mathrm{~m}^{3} \mathrm{ha}^{-1}$ para la mayor y menor densidad, respectivamente; pero sin llegar a alcanzar el estimado en $152.7 \mathrm{~m}^{3} \mathrm{ha}^{-1}$ para la plantación de teca en general (Fig. 3).

Con respecto al efecto del sitio de plantación sobre el volumen, comparativamente, el sitio II presentó la mayor tasa de crecimiento, a la edad de rotación (19 años) se estiman $195.1 \mathrm{~m}^{3} \mathrm{ha}^{-1}$ en la mejor calidad de sitio (IS $=18$ m) (Fig. 3d). El segundo mejor sitio de plantación fue el I, que a la edad e IS indicados presenta un rendimiento de $166.8 \mathrm{~m}^{3} \mathrm{ha}^{-1}$. En tanto, en el sitio III, bajo las mismas condiciones, se estiman $152.6 \mathrm{~m}^{3} \mathrm{ha}^{-1}$. Respecto al sitio IV, se considera que el volumen se subestima, debido al reducido intervalo de edad (11 meses a 56 meses) de las observaciones trabajadas. En ese sentido, para los sistemas de crecimiento en área basal y volumen seleccionados, la incorporación de nuevas observaciones a mayores edades de la plantación, sobre todo de los sitios más jóvenes, se podría verificar y en su caso rectificar el efecto de densidad y de sitio de plantación tal y como lo sugieren FierrosMateo et al. (2017), lo que permitiría reajustar la tendencia que hasta el momento predicen los modelos específicos y que tienden a ser conservadores. 

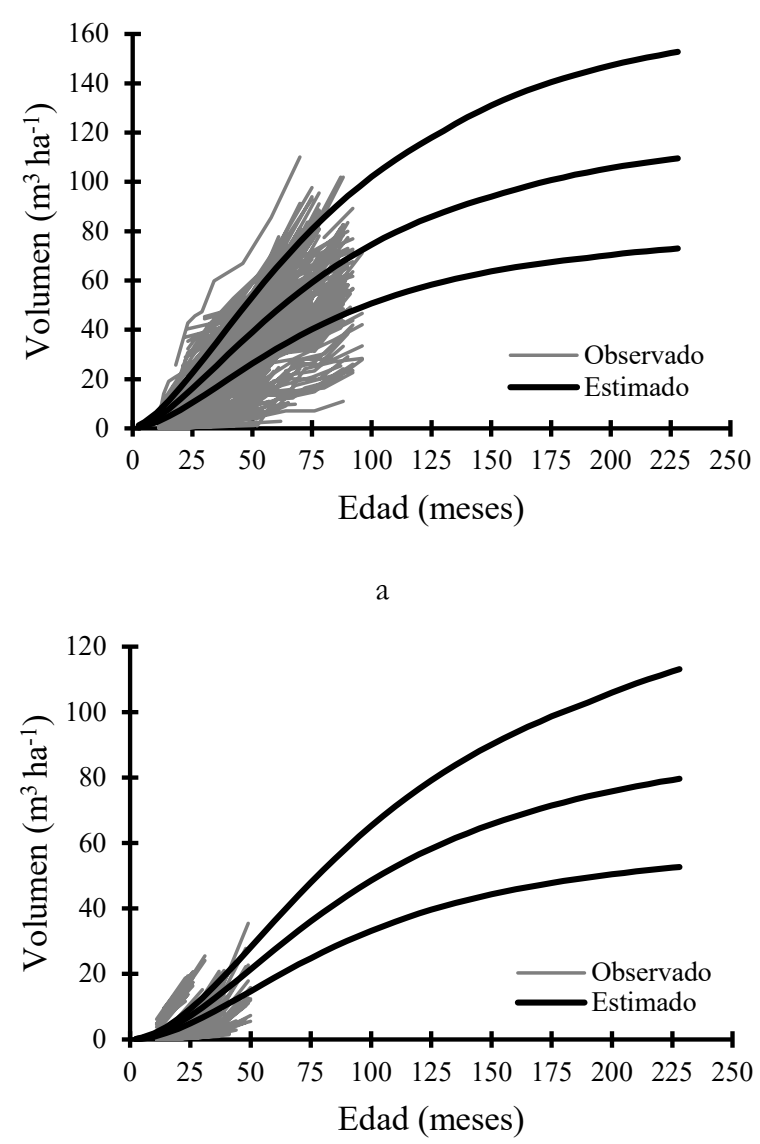

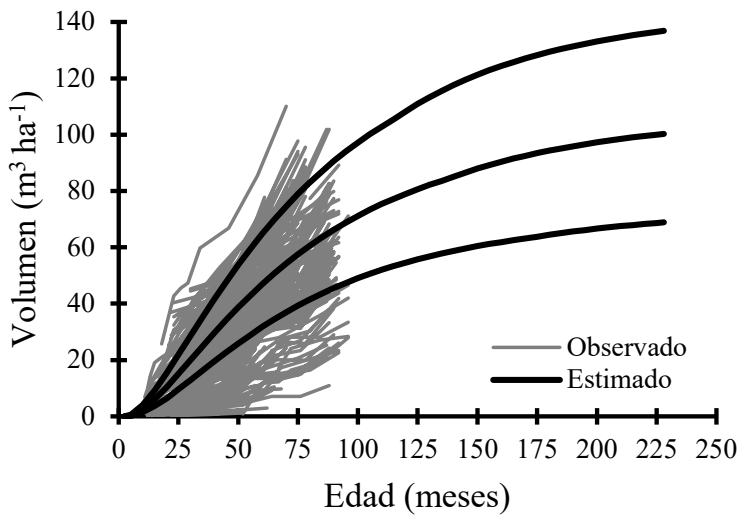

b

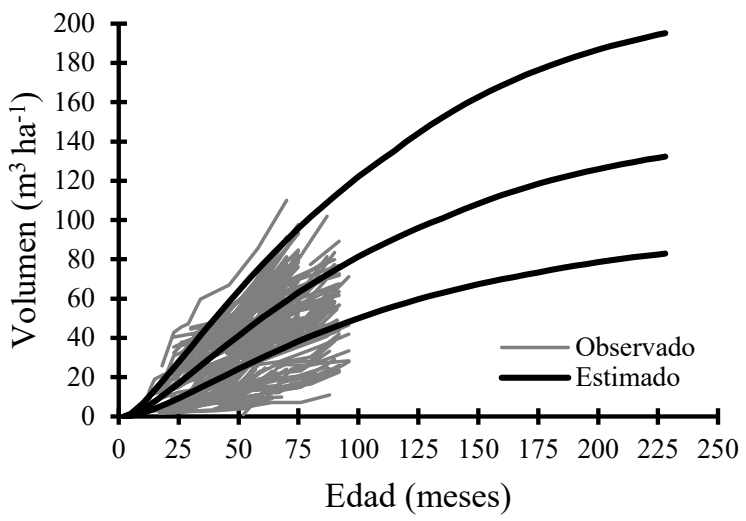

d

FIGURA 3. Crecimiento del volumen de tres índices de sitio (IS) $\left(12 \mathrm{~m}, 15 \mathrm{~m} \mathrm{y} 18 \mathrm{~m}\right.$ ) para el Scrm general (a), para $\mathrm{N}=1250 \mathrm{pl} \mathrm{ha}{ }^{-1}$ (b), para $\mathrm{N}=816 \mathrm{pl} \mathrm{ha}^{-1}$ (c) y para el sitio de plantación II con $\mathrm{N}=1250 \mathrm{pl} \mathrm{ha}^{-1}$ (d).

Cada componente del Scrm se construyó sobre una base teórica con significado biológico, por lo que las estimaciones que el sistema realiza fuera del intervalo de edad utilizado para su construcción describen un patrón de crecimiento razonable y confiable. En este sentido, Huang, Yang y Wang (2003) indican que cuando la elección de los modelos se basa en supuestos biológicos, estos pueden funcionar bien al hacer extrapolaciones. El Scrm generado representa una aproximación a la condición promedio de la tendencia biológica de las plantaciones estudiadas, por lo que puede utilizarse como una herramienta silvícola de soporte y apoyo a la toma de decisiones para su manejo. En la construcción del sistema se trabajó con un intervalo de edad relativamente corto comparado con el turno; sin embargo, aunque ofrece estimaciones conservadoras, mantiene tendencias y comportamientos gráficos probables.

El cumplimiento de los supuestos de regresión (homocedasticidad y no autocorrelación en los términos de error) para los modelos del sistema S4 de crecimiento en volumen, garantiza que los estimadores son insesgados, consistentes y eficientes, por lo que se pueden obtener intervalos de predicción realistas y efectuar pruebas de hipótesis correctas (Parresol, 2001). Esto se atribuye a que en la generación tanto del modelo de volumen fustal como de la ecuación de altura dominante e IS, usados para generar el Scrm, Tamarit et al., (2014a) y Tamarit et al., (2014b) realizaron apropiadamente las correcciones de heterocedasticidad y autocorrelación. 


\section{CONCLUSIONES}

El sistema de crecimiento y rendimiento maderable explícito generado con base en ecuaciones dinámicas permite estimar el volumen total con corteza a nivel de hectárea bajo condiciones locales específicas de establecimiento, manejo y de calidad de sitio de las plantaciones de teca establecidas en Campeche, México.

La determinación puede realizarse a nivel de toda la plantación en general o bien por densidad de plantación inicial $\left(1250 \mathrm{pl} \mathrm{ha}^{-1}\right.$ y $816 \mathrm{pl} \mathrm{ha-1}$ ) y por sitio de plantación para la mayor densidad; incluso con las ecuaciones dinámicas del Scrm y conociendo el índice de sitio particular, es posible construir curvas de crecimiento locales para cada lote de manejo específico en que se tiene clasificada a la PFC.

Con un índice de sitio de $18 \mathrm{~m}$ (edad de referencia de 7.5 años) clasificado como el más alto, para toda la plantación se estiman $159.8 \mathrm{~m}^{3} \mathrm{ha}^{-1}$, en promedio, al turno de 19 años. Para la densidad inicial de $1250 \mathrm{pl} \mathrm{ha}^{-1}$ y el índice de sitio indicado, se determinó que el sitio de plantación identificado como II fue el más productivo, estimándose en promedio $196.6 \mathrm{~m}^{3} \mathrm{ha}^{-1}$ a la edad del turno.

\section{REFERENCIAS}

Álvarez-González, J. G., Castedo-Dorado, F., Ruiz-González, A. D., López-Sánchez, C. A., \& Gadow, K. (2004). A two-step mortality model for evenaged stands for Pinus radiata D, Don in Galicia (Northwestern Spain). Annals of Forest Science, 61, 439-448. doi: 10.1051/forest:2004037

Bermejo, I., Cañellas I., \& San Miguel, A. (2004). Growth and yield models for teak plantations in Costa Rica. Forest Ecology and Management, 189, 97-110. doi: 10.1016/j.foreco.2003.07.031

Breña P., A. F. (2004). Precipitación y recursos hidráulicos en México. México, D. F: Universidad Autónoma Metropolitana.

Camacho-Linton, A., Ramírez-Maldonado, H., De los Santos-Posadas, H. M., \& Zamudio S., F. J. (2013). Tablas de rendimiento para teca (Tectona grandis L.) en el estado de Campeche. Revista Mexicana de Ciencias Forestales, 4(19), 92-101.

Castedo D., F., Barrio A., M., Parresol, B. R., \& Álvarez G., J. G. (2005). A stochastic height-diameter model for maritime pine ecoregions in Galicia (northwestern Spain). Annals of Forest Science, 62, 455465. doi: 10.1051 / forest:2005042
Clutter, J. L., Fortson, J. C., Pienaar, L. V., Brister, G. H., \& Bailey, R. L. (1983). Timber management: a quantitative approach. New York, USA: John Wiley \& Sons.

Comisión Nacional Forestal [Conafor]. (2014). Principales especies maderables establecidas en PFC por Entidad Federativa en 2000 - 2014. Recuperado de: http://www.conafor.gob.mx:8080/documentos/docs/43/6019 Principales especies maderables establecidas en PFC por Entidad Federativa en 2000 - 2014.pdf

Diéguez-Aranda, U., Castedo-Dorado, F., Álvarez-González, J. G., \& Rodríguez-Soalleiro, R. (2005). Modelling mortality of Scots pine (Pinus sylvestris L.) plantations in the northwest of Spain. European Journal of Forest Research, 124, 143-153. doi: 10.1007/s10342-004-0043-5

Draper, N. R., \& Smith, H. 1998. Applied regression analysis (3a. ed.). Nueva Jersey, EUA: John Wiley \& Sons.

Food and Agriculture Organization of the United Nations [FAO]. (2010). Global forest resources assessment 2010. Forestry Paper 163. Rome, Italy. Recuperado de http://www.fao.org/3/a-i1757e.pdf

Fierros-Mateo, R., De los Santos-Posadas, H. M., Fierros-González, M. A., \& Cruz-Cobos, F. (2017). Crecimiento y rendimiento maderable en plantaciones de Pinus chiapensis (Martínez) Andresen. Agrociencia, 51, 201-214.

Hallett, J. T., Díaz-Calvo, J., Villa-Castillo, J., \& Wagner, M. R. (2011). Teak plantations: economic bonanza or environmental disaster? Journal of Forestry, 109, 288-292. doi: 10.1093/jof/109.5.288

Hirigoyen, A., Franco, J., \& Diéguez, U. 2018. Modelo dinámico de rodal para Eucalyptus globulus (L.) en Uruguay. Agrociencia Uruguay, 22(1), 63-80. doi: 10.31285/agro.22.1.7

Huang, S., Yang, Y., \& Wang, Y. (2003). A critical look at procedures for validating growth and yield models. En: Amaro A., Reed D. and Soares, P. (Eds.). Modelling Forest Systems (pp. 271-293). Wallingford, Oxfordshire, UK: CAB International.

Kollert, W., \& Cherubini, L. (2012). Teak resources and market assessment 2010 (Tectona grandis Linn. F.). Working Paper FP/47/E. FAO. Rome, Italy. Recuperado de http://www.fao.org/3/a-an537e.pdf

Návar, J., Dominguez-Calleros, P. A., Rodriguez-Flores, F. J., LizárragaMendiola, L., de Hoogh, R., \& Synnott, J. T. (2016). A stand growth and yield model for northern reforested stands of Mexico. Mathematical and Computational Forestry \& Natural-Resource Sciences, 8(1), 25-34. Recuperado de https://pdfs.semanticscholar.org/eb00/e5c444f5c2abe203ec066 88e2b3fbab94e11.pdf

Nunifu, T. K., \& Murchison, H. G. (1999). Provisional yield models of teak (Tectona grandis Linn F.) plantations in northern Ghana. Forest 
Ecology and Management, 120, 171-178. doi: 10.1016/S03781127(98)00529-5

Parra-Piedra, J. P., De los Santos-Posadas H. M., Fierros-González, A. M., Valdez-Lazalde, J. R., \& Romo-Lozano, J. L. (2017). Proyección explícita e implícita del rendimiento maderable de plantaciones forestales comerciales de Pinus patula Schiede ex Schltdl. et Cham. Agrociencia, 51, 455-470.

Parresol, B. R. (2001). Additivity of nonlinear biomass equations. Canadian Journal of Forest Research, 31(5), 865-878. doi: 10.1139/x00-202

Pereira, da C. J., Garcia, L. H., Boechat, S. C. P., Chagas, C. J. C., Smit, L., Saraiva, N. G., \& Romarco, de O. M. L. (2008). Modelos de crescimento e produção para plantios comerciais jovens de Tectona grandis em Tangará da Serra, Mato Grosso. Árvore, 32, 821-828.

Pérez, D., \& Kanninen, M. (2005). Stand growth scenarios for Tectona grandis plantations in Costa Rica. Forest Ecology and Management, 210, 425-441. doi: 10.1016/j.foreco.2005.02.037

Pretzsch, H. (2009). Forest dynamics, growth and yield. From measurement to model. Berlin, Germany: Springer-Verlag.

Restrepo O., H. I., Orrego S., S. A., del Valle A., J. I., \& Salazar U., J. C. (2012). Rendimiento, turno óptimo forestal y rentabilidad de plantaciones forestales de Tectona grandis y Pinus patula en Colombia. Interciencia, 37, 14-20.

Rose Jr., Ch. E., Clutter, M. L., Shiver, B. D., Hall, D. B., \& Borders, B. (2004). A generalized methodology for developing whole-stand survival models. Forest Science, 50, 686-695. doi: 10.1093/forestscience/50.5.686

Rose Jr., Ch. E., \& Lynch, T. B. (2001). Estimating parameters for tree basal area growth with a system of equations and seemingly unrelated regressions. Forest Ecology and Management, 148, 51-61. doi: 10.1016/S0378-1127(00)00524-7

Santiago-García, W., De los Santos-Posadas, H. M., Ángeles-Pérez, G., Valdez-Lazalde, J. R., Corral-Rivas, J. J., Rodríguez-Ortiz, G., \& Santiago-García, E. (2015). Modelos de crecimiento y rendimiento de totalidad del rodal para Pinus patula. Madera $y$ Bosques, 21(3), 95-110. doi: 10.21829/myb.2015.213459

SAS Institute Inc. (2011). SAS/STAT ${ }^{\circledR} 9.2$ User's Guide. Cary, NC USA: SAS Institute Inc.

Schumacher, F. X. (1939). A new growth function curve and its application to timber-yield studies. Journal of Forestry, 37, 819-820.

Steel, R. G. D., \& Torrie, H. J. 1997. Bioestadística: Principios y procedimientos (2a ed.). Distrito Federal, México: Interamericana de México SA de CV.
Sunanda, C., \& Jayaraman, K. (2006). Prediction of stand attributes of even-aged teak stands using multilevel models. Forest Ecology and Management, 236, 1-11. doi: 10.1016/j.foreco.2006.05.039

Tamarit U., J. C., De los Santos, P. H. M., Aldrete, A., Valdez, L. J. R., Ramírez, M. H., \& Guerra, D. V. (2014a). Sistema de cubicación para árboles individuales de Tectona grandis L. f. mediante funciones compatibles de ahusamiento-volumen. Revista Mexicana de Ciencias Forestales, 5(21), 58-74.

Tamarit U., J. C., De los Santos, P. H. M., Aldrete, A., Valdez, L. J. R., Ramírez, M. H., \& Guerra, D. V. (2014b). Ecuaciones dinámicas de índice de sitio para Tectona grandis en Campeche, México. Agrociencia, 48, 225-238.

Telles A., R., Gómez C., M., Alanís R., E., Aguirre C., O. A., \& Jiménez P., J. (2018). Ajuste y selección de modelos matemáticos para predecir el volumen total fustal de Tectona grandis en Nuevo Urecho, Michoacán, México. Madera y Bosques, 24(3). doi: $10.21829 /$ myb.2018.2431544

Torres R., J. M., \& Magaña T., O. S. (2005). Evaluación de plantaciones forestales (1a ed.). Distrito Federal, México: Limusa.

Weiskittel, A. R., Hann, D. W., Kershaw, J. A., \& Vanclay, J. K. (2011). Forest growth and yield modelling. West Sussex, UK: John Wiley \&Sons.

Zellner, A. 1962. An efficient method of estimating seemingly unrelated regressions and test for aggregation bias. Journal of the American Statistical Association, 57(298), 348-368. doi: 10.2307/2281644

Zhao, D., Borders, B., Wang, M., \& Kane, M. (2007). Modeling mortality of second-rotation loblolly pine plantations in the Piedmont/Upper Coastal Plain and Lower Coastal Plain of the southern United States. Forest Ecology and Management, 252, 132 143. doi: $10.1016 /$ j.foreco. 2007.06 .030

Manuscrito recibido el 2 de enero de 2019

Aceptado el 15 de marzo de 2019

Publicado el 13 de diciembre de 2019

Este documento se debe citar como:

Tamarit-Urias, J. C., De los Santos-Posadas, H. M., Aldrete, A., Valdez-Lazalde. J. R., Ramírez-Maldonado, H., \& Guerra-De la Cruz, V. (2019). Sistema de crecimiento y rendimiento maderable para plantaciones de teca (Tectona grandis L. f.) en Campeche, México. Madera y Bosques, 25(3), e2531908. doi: 10.21829/myb.2019.2531908

Madera y Bosques por Instituto de Ecología, A.C. se distribuye bajo una Licencia Creative Commons Atribución-NoComercialCompartirlgual 4.0 Internacional. 